Article

\title{
On the stability of radical septic functional equations
}

\author{
Emanuel Guariglia ${ }^{1, *}(\mathbb{C})$ and Kandhasamy Tamilvanan ${ }^{2}$ (]) \\ 1 Institute of Biosciences, Letters and Exact Sciences, São Paulo State University (UNESP), São José do Rio Preto, \\ SP 15054-000, Brazil \\ 2 Department of Mathematics, Government Arts College for Men, Krishnagiri, Tamil Nadu 635 001, India; \\ tamiltamilk7@gmail.com \\ * Correspondence: emanuel.guariglia@gmail.com; emanuel.guariglia@unesp.br
}

Received: 20 September 2020; Accepted: 4 November 2020; Published: 16 December 2020

check for updates

Abstract: This paper deals with the approximate solution of the following functional equation

$$
f\left(\sqrt[7]{x^{7}+y^{7}}\right)=f(x)+f(y)
$$

where $f$ is a mapping from $\mathbb{R}$ into a normed vector space. We show stability results of this equation in quasi- $\beta$-Banach spaces and $(\beta, p)$-Banach spaces. We also prove the nonstability of the previous functional equation in a relevant case.

Keywords: radical functional equation; septic functional equation; Hyers-Ulam stability; quasi- $\beta$-Banach spaces; $(\beta, p)$-Banach spaces

MSC: 39B52; 39B82; 46H25

\section{Introduction and preliminaries}

The stability problem of functional equations originated from a question of Ulam [1] in 1940 for the Cauchy equation

$$
f(x+y)=f(x)+f(y) .
$$

Each solution of (1) is called additive. This problem was partially solved by Hyers [2] in 1941. Some years later, Aoki [3] and Bourgin [4] treated the stability problem with unbounded Cauchy difference. In 1978, Th.M. Rassias [5] thus provided a generalization of the Hyers theorem for unbounded Cauchy differences. On the other hand, Rassias [6] dealt with the case of Cauchy differences controlled by a product of different powers of norms. Likewise, Forti [7] and Găvruţa [8] generalized these results to arbitrary unbounded Cauchy differences. Hyers-Ulam stability for the linear functional equation in single variable is, in a clear way and in detail, treated in [9]. We also mention the papers [10-12] concerning the application of different fixed point theorems to the theory of Hyers-Ulam stability.

It is easy to see that the functional equation

$$
f(x+y)+f(x-y)=2 f(x)+2 f(y),
$$

admits as a solution the function $f(x)=c x^{2}$, where $c$ is an arbitrary constant. Accordingly, Eq. (2) is called quadratic functional equation. In the same spirit, solutions of (2) are called quadratic functions. Likewise, we can built function $f(x)=c x^{m}$ with $m \in \mathbb{N} \mid m>2$ as solutions of a linear functional equations in the 
same spirit of (2). These functional equations are called cubic, quartic, quintic and so on (see [13-15] for more details). In particular, Shen and Chen [16] studied the general solutions of the functional equation

$$
\begin{gathered}
f(x+4 y)-7 f(x+3 y)+21 f(x+2 y)-35 f(x+y)+35 f(x)-21 f(x-y) \\
+7 f(x-2 y)-f(x-3 y)=5040 f(y),
\end{gathered}
$$

on commutative groups. Note that the function $f(x)=c x^{7}$ satisfies (3), thus Eq. (3) is a septic functional equation. Accordingly, each solution of Eq. (3) is called a septic function.

Quite recently, considerable attention has been paid to the stability of radical function equations (see, e.g., [17-19]). More precisely, the functional equation

$$
f\left(\sqrt{x^{2}+y^{2}}\right)=f(x)+f(y)
$$

$f$ being a mapping from $\mathbb{R}$ into a normed vector space, is called a radical quadratic functional equation. Kim et al. [20,21] studied the following generalizations of Eq. (4)

$$
\begin{gathered}
f\left(\sqrt{a x^{2}+b y^{2}}\right)=a f(x)+b f(y), \\
f\left(\sqrt{a x^{2}+b y^{2}}\right)+f\left(\sqrt{\left|a x^{2}-b y^{2}\right|}\right)=2 a^{2} f(x)+2 b^{2} f(y),
\end{gathered}
$$

where $a, b \in \mathbb{R}_{\geq 0}$. The same authors proved the generalized Ulam stability for (5) and (6), similar in spirit to Găvruţa [8]. In particular, the authors generalized the concept of Ulam stability by more general mappings. Moreover, Cho et al. [22] proved the generalized Hyers-Ulam stability for (5) and (6) both in quasi- $\beta$-Banach spaces and $(\beta, p)$-Banach spaces.

Ding and $\mathrm{Xu}$ [23] provided a further generalization of (4) by the following inhomogeneous functional equation

$$
f\left(\sqrt{a x^{2}+b y^{2}}\right)=a f(x)+b f(y)+D(x, y),
$$

where $f$ is a mapping on the set of real numbers, $a, b \in \mathbb{R}_{\geq 0}$ and $D(x, y)$ is a given function. Furthermore, Ding and $\mathrm{Xu}$ showed stability and hyperstability properties for Eq. (7) by Brzdek and Cieplinski's fixed point theorems in 2-Banach spaces.

We note that Eq. (4) allows a natural and straightforward generalization as follows:

$$
f\left(\sqrt[n]{x^{n}+y^{n}}\right)=f(x)+f(y)
$$

$f$ being a mapping from $\mathbb{R}$ into a normed vector space and $n \in \mathbb{N} \mid n \geq 2$. In last years, several authors dealt with the cases $n=3,4,5,6$ in Eq. (8). For more details we refer the reader to [24-27]. In particular, EL-Fassi treated the case $n=5$ providing stability results in quasi- $\beta$-Banach spaces.

In this paper, we consider the following functional equation:

$$
f\left(\sqrt[7]{x^{7}+y^{7}}\right)=f(x)+f(y)
$$

$f$ being a mapping from $\mathbb{R}$ into a normed vector space. We prove that Eq. (9) is septic. Furthermore, we treat the Hyers-Ulam stability for (9) in quasi- $\beta$-Banach spaces and $(\beta, p)$-Banach spaces. Our results have many potential applications in information theory, dynamical systems, computer graphics, etc. (see, e.g., [28-31]). 
Let us recall the definition of quasi- $\beta$-Banach space and $(\beta, p)$-Banach space because in the sequel we will deal with the stability of (9) in these function spaces.

Definition 1 ([24,25]). Fix a real number $\beta$ with $0<\beta \leq 1$. Let $\mathbb{F}$ denote either $\mathbb{R}$ or $\mathbb{C}$ and let $X$ be a vector space over $\mathbb{F}$. A quasi- $\beta$-norm on $X$ is a function $\|\cdot\|: X \rightarrow \mathbb{R}$ such that

(i) $\|x\| \geq 0$ and $\|x\|=0$ if and only if $x=0$,

(ii) $\|\lambda x\|=|\lambda|^{\beta}\|x\|$,

(iii) $\|x+y\| \leq C(\|x\|+\|y\|)$,

where $C \geq 1$ is a constant, for all $x, y \in X$ and $\lambda \in \mathbb{F}$.

Note that in Definition 1 the constant $C$ is independent of $x, y \in X$. The pair $(X,\|\cdot\|)$ is called a quasi- $\beta$-normed space if $\|\cdot\|$ is a quasi- $\beta$-norm on $X$. The smallest constant $C$ is called modulus of concavity of $\|\cdot\|$. A quasi- $\beta$-norm induces a locally bounded topology on $X$ and conversely (see [32] for more details). A complete quasi- $\beta$-normed space is called a quasi- $\beta$-Banach space. If, in addition, we have

$$
\|x+y\|^{p} \leq\|x\|^{p}+\|y\|^{p}, \quad 0<p \leq 1, \text { for all } x, y \in X,
$$

then a quasi- $\beta$-norm $\|\cdot\|$ is called a $(\beta, p)$-norm. A quasi- $\beta$-Banach space $(X,\|\cdot\|)$ is thus called a $(\beta, p)$-Banach space if $\|\cdot\|$ is a $(\beta, p)$-norm on $X$.

Remark 1. Definition 1 and (10) show that quasi- $\beta$-Banach spaces and $(\beta, p)$-Banach spaces are a generalization of quasi-Banach spaces and $p$-Banach spaces [32,33], respectively.

Definition 1 for $\beta=1$ gives the usual concepts of quasi-norm and $p$-norm. Of course, any $p$-norm is a quasi-norm with $C=2^{1 / p-1}$. Up to equivalence, the converse is true and called the Aoki-Rolewicz theorem $[4,34]$.

\section{Solution of Eq. (9)}

Our aim in this section is to find the general solution of Eq. (9). This is relevant to deal with the stability of Eq. (9). In order to get this result, we simply proceed by induction. Here and subsequently, $\mathbb{Z}^{*}$ denotes the set of nonzero integers and $\mathbb{N}_{0}=\mathbb{N} \cup\{0\}$.

Theorem 1. Let $V$ be a real vector space and $f: \mathbb{R} \rightarrow V$ be a function which fulfils Eq. (9). Then $f$ is a septic function.

Proof. By choosing $x=y=0$ in (9), we see at once that $f(0)=0$. Replacing $y=-x$ in (9), we obtain $f(-x)=-f(x)$ for all $x \in \mathbb{R}$. Likewise, replacing $y=x$ in (9), we have that $f(\sqrt[7]{2} x)=2 f(x)$ for all $x \in \mathbb{R}$. Setting $y=\sqrt[7]{2} x$ in (9), the equality $f(\sqrt[7]{2} x)=2 f(x)$ implies that $f(\sqrt[7]{3} x)=3 f(x)$ for all $x \in \mathbb{R}$.

By induction, we get $f(\sqrt[7]{k} x)=k f(x)$ for all $x \in \mathbb{R}$ and $k \in \mathbb{Z}$. Accordingly,

$$
f\left(\frac{x}{\sqrt[7]{k}}\right)=\frac{f(x)}{k} \Rightarrow f\left(\sqrt[7]{\frac{m}{k}} x\right)=\frac{m}{k} f(x),
$$

for all $x \in \mathbb{R}$ and $k, m \in \mathbb{Z}^{*}$. Therefore,

$$
f(\sqrt[7]{r} x)=r f(x)
$$


for all $x \in \mathbb{R}$ and $r \in Q$. The proof is completed by showing that the function $f$ fulfils Eq. (3). In fact, from (9) and (11) we get

$$
\begin{aligned}
& f(x+4 y)+21 f(x+2 y)+35 f(x)+7 f(x-2 y) \\
& =f(x+4 y)+f(\sqrt[7]{21}(x+2 y))+f(\sqrt[7]{35} x)+f(\sqrt[7]{7}(x-2 y)) \\
& =f\left(\sqrt[7]{(x+4 y)^{7}+21(x+2 y)^{7}+35 x^{7}+7(x-2 y)^{7}}\right)
\end{aligned}
$$

and similarly

$$
\begin{aligned}
& 7 f(x+3 y)+35 f(x+y)+21 f(x-y)+f(x-3 y)+5040 f(y) \\
& =f\left(\sqrt[7]{7(x+3 y)^{7}+35(x+y)^{7}+21(x-y)^{7}+(x-3 y)^{7}+5050 y^{7}}\right)
\end{aligned}
$$

for all $x, y \in \mathbb{R}$. Comparing (12) and (13) implies that $f$ is septic, as desired.

\section{Hyers-Ulam stability of Eq. (9) in quasi- $\beta$-Banach spaces}

This section is devoted to the Hyers-Ulam stability of Eq. (9) in quasi- $\beta$-Banach spaces. In the same spirit of [24], we introduce the definition of $\gamma$-approximately radical septic function.

Let $X$ be a quasi- $\beta$-Banach space. A $\gamma$-approximately radical septic function is a function $f: \mathbb{R} \rightarrow X$ such that

$$
\left\|f\left(\sqrt[7]{x^{7}+y^{7}}\right)-f(x)-f(y)\right\| \leq \gamma(x, y),
$$

for all $x, y \in \mathbb{R}$ and where $\gamma$ is a nonnegative real function on $\mathbb{R}^{2}$.

Theorem 2. Let $X$ be a quasi- $\beta$-Banach space. Moreover, let $f$ be a $\gamma$-approximately radical septic function and $\Gamma: \mathbb{R}^{2} \rightarrow[0, \infty)$ be a function such that

$$
\Gamma(x, y):=\sum_{i=0}^{\infty}\left(\frac{C}{2^{\beta}}\right)^{i} \gamma\left(\sqrt[7]{2^{i}} x, \sqrt[7]{2^{i}} y\right)<\infty,
$$

and

$$
\frac{\gamma\left(\sqrt[7]{2^{k}} x, \sqrt[7]{2^{k}} y\right)}{2^{k \beta}} \stackrel{k \rightarrow \infty}{\longrightarrow} 0,
$$

for all $x, y \in \mathbb{R}$. Then Eq. (9) has a unique septic solution $L: \mathbb{R} \rightarrow X$ satisfying

$$
\|f(x)-L(x)\| \leq \frac{C}{2^{\beta}} \Gamma(x, x),
$$

for all $x \in \mathbb{R}$.

Proof. We divide the proof into two parts (existence and uniqueness). Inequality (14) for $y=x$ gives

$$
\|f(\sqrt[7]{2} x)-2 f(x)\| \leq \gamma(x, x),
$$

and therefore

$$
\left\|\frac{f(\sqrt[7]{2} x)}{2}-f(x)\right\| \leq \frac{\gamma(x, x)}{2^{\beta}}
$$


for all $x \in \mathbb{R}$. We have

$$
\begin{aligned}
\left\|\frac{f\left(\sqrt[7]{2^{l}} x\right)}{2^{l}}-\frac{f\left(\sqrt[7]{2^{m}} x\right)}{2^{m}}\right\| & =\left\|\sum_{i=l}^{m-1}\left(\frac{f\left(\sqrt[7]{2^{i}} x\right)}{2^{i}}-\frac{f\left(\sqrt[7]{2^{i+1}} x\right)}{2^{i+1}}\right)\right\| \\
& \leq \sum_{i=l}^{m-1} \frac{1}{2^{\beta i}}\left\|f\left(\sqrt[7]{2^{i} x}\right)-\frac{f\left(\sqrt[7]{2^{i+1}} x\right)}{2}\right\| \\
& \stackrel{(18)}{\leq} \frac{C}{2^{\beta}} \sum_{i=l}^{m-1}\left(\frac{C}{2^{\beta}}\right)^{i} \gamma\left(\sqrt[7]{2^{i}} x, \sqrt[7]{2^{i}} x\right), \quad m, l \in \mathbb{N}_{0}, m>l
\end{aligned}
$$

for all $x \in \mathbb{R}$. Note that (15) and (19) entail that $\left\{2^{-k} f\left(\sqrt[7]{2^{k}} x\right)\right\}$ is a Cauchy sequence on the whole real line. Moreover, $X$ is quasi- $\beta$-Banach space thus the previous sequence converges over the entire real line. This allows us to define a function $L: \mathbb{R} \rightarrow X$ by

$$
L(x):=\lim _{k \rightarrow \infty} \frac{f\left(\sqrt[7]{2^{k}} x\right)}{2^{k}}
$$

for all $x \in \mathbb{R}$. We thus get

$$
\begin{aligned}
\left\|L\left(\sqrt[7]{x^{7}+y^{7}}\right)-L(x)-L(y)\right\| & =\lim _{k \rightarrow \infty} 2^{-\beta k}\left\|f\left(\sqrt[7]{2^{k}} \sqrt[7]{x^{7}+y^{7}}\right)-f\left(\sqrt[7]{2^{k}} x\right)-f\left(\sqrt[7]{2^{k}} y\right)\right\| \\
& \leq \lim _{k \rightarrow \infty} 2^{-\beta k} \gamma\left(\sqrt[7]{2^{k}} x, \sqrt[7]{2^{k}} y\right)=0
\end{aligned}
$$

and so

$$
L\left(\sqrt[7]{x^{7}+y^{7}}\right)=L(x)+L(y)
$$

for all $x, y \in \mathbb{R}$. From (20), we deduce that $L$ is a septic function. In addition to this, letting $m \rightarrow \infty$ in (19) with $l=0$ we conclude that $L$ satisfies (16) near $f$.

We are now in a position to show the uniqueness of $L$. Hence, let us consider a septic function $S: \mathbb{R} \rightarrow X$ which also satisfies both (9) and (16). Since $S$ is a solution of (9), it follows that $S\left(\sqrt[7]{2^{k}} x\right)=2^{k} S(x)$ for all $x \in \mathbb{R}$ and $k \in \mathbb{N}_{0}$. As a consequence,

$$
\begin{aligned}
\left\|\frac{f\left(\sqrt[7]{2^{k}} x\right)}{2^{k}}-S(x)\right\| & =2^{-k \beta}\left\|f\left(\sqrt[7]{2^{k}} x\right)-S\left(\sqrt[7]{2^{k}} x\right)\right\| \\
& \leq \frac{C}{2^{(k+1) \beta}} \Gamma\left(\sqrt[7]{2^{k}} x, \sqrt[7]{2^{k}} x\right), \quad x \in \mathbb{R} .
\end{aligned}
$$

which for $k \rightarrow \infty$ gives $S(x)=L(x)$ for all $x \in \mathbb{R}$. The proof is achieved.

In a similar way we obtain the following result.

Theorem 3. Let $X$ be a quasi- $\beta$-Banach space. Moreover, let $f$ be a $\gamma$-approximately radical septic function and $\Lambda: \mathbb{R}^{2} \rightarrow[0, \infty)$ be a function such that

$$
\Lambda(x, y):=\sum_{i=0}^{\infty}\left(2^{\beta} C\right)^{i} \gamma\left(\frac{x}{\sqrt[7]{2^{i+1}}}, \frac{y}{\sqrt[7]{2^{i+1}}}\right)<\infty
$$


and

$$
2^{k \beta} \gamma\left(\frac{x}{\sqrt[7]{2^{k}}}, \frac{y}{\sqrt[7]{2^{k}}}\right) \stackrel{k \rightarrow \infty}{\longrightarrow} 0
$$

for all $x, y \in \mathbb{R}$. Then Eq. (9) has a unique septic solution $L: \mathbb{R} \rightarrow X$ satisfying

$$
\|f(x)-L(x)\| \leq C \Lambda(x, x)
$$

for all $x \in \mathbb{R}$.

Proof. Replacing $x$ by $\frac{x}{\sqrt[7]{2}}$ in (17), the proof runs as in Theorem 2. For the sake of completeness, we only sketch the proof here thus leaving the details to the reader.

Clearly,

$$
\left\|f(x)-2 f\left(\frac{x}{\sqrt[7]{2}}\right)\right\| \leq \gamma\left(\frac{x}{\sqrt[7]{2}}, \frac{x}{\sqrt[7]{2}}\right) .
$$

Therefore, we have

$$
\begin{aligned}
\left\|2^{l} f\left(\frac{x}{\sqrt[7]{2^{l}}}\right)-2^{m} f\left(\frac{x}{\sqrt[7]{2^{m}}}\right)\right\| & =\left\|\sum_{i=l}^{m-1}\left(2^{i} f\left(\frac{x}{\sqrt[7]{2^{i}}}\right)-2^{i+1} f\left(\frac{x}{\sqrt[7]{2^{i+1}}}\right)\right)\right\| \\
& \leq \sum_{i=l}^{m-1} 2^{\beta i}\left\|f\left(\frac{x}{\sqrt[7]{2^{i}}}\right)-2 f\left(\frac{x}{\sqrt[7]{2^{i+1}}}\right)\right\| \\
& \stackrel{(22))}{\leq} C \sum_{i=l}^{m-1}\left(2^{\beta} C\right)^{i} \gamma\left(\frac{x}{\sqrt[7]{2^{i+1}}}, \frac{x}{\sqrt[7]{2^{i+1}}}\right), \quad m, l \in \mathbb{N}_{0}: m>l,
\end{aligned}
$$

for all $x \in \mathbb{R}$. As a consequence, the sequence $\left\{2^{k} f\left(\frac{x}{\sqrt[7]{2^{k}}}\right)\right\}$ converges over the entire real line. This allows us to define a function $L: \mathbb{R} \rightarrow X$ by

$$
L(x):=\lim _{k \rightarrow \infty} 2^{k} f\left(\frac{x}{\sqrt[7]{2^{k}}}\right)
$$

for all $x \in \mathbb{R}$. Hence

$$
L\left(\sqrt[7]{x^{7}+y^{7}}\right)=L(x)+L(y)
$$

for all $x \in \mathbb{R}$. This prove the existence of the solution $L$ satisfying both (9) and (21). Now, let us consider a septic function $S: \mathbb{R} \rightarrow X$ which also satisfies both (9) and (21). The uniqueness of $L$ follows noting that $S\left(\frac{x}{\sqrt[7]{2^{k}}}\right)=2^{-k} S(x)$ for all $x \in \mathbb{R}$ and $k \in \mathbb{N}_{0}$.

Theorems 2 and 3 imply the stability for approximate functions controlled by powers of norms, as stated in the following two corollaries.

Corollary 1. Let $X$ be a quasi- $\beta$-Banach space. Moreover, let $f: \mathbb{R} \rightarrow X$ be a function such that

$$
\left\|f\left(\sqrt[7]{x^{7}+y^{7}}\right)-f(x)-f(y)\right\| \leq \begin{cases}\epsilon\left(|x|^{r}|y|^{s}\right), & r+s<7\left(\beta-\log _{2} C\right), \\ \epsilon\left(|x|^{r}+|y|^{s}\right), & r, s<7\left(\beta-\log _{2} C\right), \\ \epsilon\left(|x|^{r}|y|^{s}+|x|^{r+s}+|y|^{r+s}\right), & r+s<7\left(\beta-\log _{2} C\right),\end{cases}
$$


for all $x, y \in \mathbb{R}$, with $r, s, \in \in \mathbb{R}_{\geq 0}$. Then $E q$. (9) has a unique septic solution $L: \mathbb{R} \rightarrow X$ satisfying

$$
\|f(x)-L(x)\| \leq \begin{cases}\frac{\epsilon C}{2^{\beta}-C \sqrt[7]{2^{r+s}}}|x|^{r+s}, & r+s<7\left(\beta-\log _{2} C\right), \\ \epsilon C\left(\frac{|x|^{r}}{2^{\beta}-C \sqrt[7]{2^{r}}}+\frac{|x|^{s}}{2^{\beta}-C \sqrt[7]{2^{s}}}\right), & r, s<7\left(\beta-\log _{2} C\right), \\ \frac{3 \epsilon C}{2^{\beta}-C \sqrt[7]{2^{r+s}}}|x|^{r+s}, & r+s<7\left(\beta-\log _{2} C\right),\end{cases}
$$

for all $x \in \mathbb{R}$.

Proof. With the same notation of Theorem 2, we have

$$
\gamma(x, y)= \begin{cases}\epsilon\left(|x|^{r}|y|^{s}\right), & r+s<7\left(\beta-\log _{2} C\right), \\ \epsilon\left(|x|^{r}+|y|^{s}\right), & r, s<7\left(\beta-\log _{2} C\right), \\ \epsilon\left(|x|^{r}|y|^{s}+|x|^{r+s}+|y|^{r+s}\right), & r+s<7\left(\beta-\log _{2} C\right) .\end{cases}
$$

for all $x, y \in \mathbb{R}$. Thus the proof falls naturally into three cases.

First case: $\gamma(x, y)=\epsilon\left(|x|^{r}|y|^{s}\right)$.

We see that

$$
\begin{aligned}
\|f(x)-L(x)\| & \leq \frac{C}{2^{\beta}} \Gamma(x, x)=\frac{C}{2^{\beta}} \sum_{i=0}^{\infty}\left(\frac{C}{2^{\beta}}\right)^{i} \gamma\left(\sqrt[7]{2^{i}} x, \sqrt[7]{2^{i}} x\right) \\
& =\frac{C}{2^{\beta}} \sum_{i=0}^{\infty}\left(\frac{C}{2^{\beta}}\right)^{i} \epsilon\left(\left|\sqrt[7]{2^{i}} x\right|^{r}\left|\sqrt[7]{2^{i}} x\right|^{s}\right) \\
& =\frac{C}{2^{\beta}} \epsilon|x|^{r+s} \sum_{i=0}^{\infty}\left(\frac{C}{2^{\beta}}\right)^{i}\left(\sqrt[7]{2^{r+s}}\right)^{i},
\end{aligned}
$$

for all $x \in \mathbb{R}$. Notice that the condition $r+s<7\left(\beta-\log _{2} C\right)$ implies the convergence of the last series in (25). Accordingly,

$$
\|f(x)-L(x)\| \leq \frac{\epsilon C}{2^{\beta}-C \sqrt[7]{2^{r+s}}}|x|^{r+s} .
$$

Second case: $\gamma(x, y)=\epsilon\left(|x|^{r}+|y|^{s}\right)$.

We have that

$$
\begin{aligned}
\|f(x)-L(x)\| & \leq \frac{C}{2^{\beta}} \Gamma(x, x)=\frac{C}{2^{\beta}} \sum_{i=0}^{\infty}\left(\frac{C}{2^{\beta}}\right)^{i} \gamma\left(\sqrt[7]{2^{i}} x, \sqrt[7]{2^{i}} x\right) \\
& =\frac{C}{2^{\beta}} \sum_{i=0}^{\infty}\left(\frac{C}{2^{\beta}}\right)^{i} \epsilon\left(\left|\sqrt[7]{2^{i} x}\right|^{r}+\left|\sqrt[7]{2^{i}} x\right|^{S}\right) \\
& =\frac{C}{2^{\beta}} \epsilon\left(|x|^{r} \sum_{i=0}^{\infty}\left(\frac{C}{2^{\beta}}\right)^{i}\left(\sqrt[7]{2^{r}}\right)^{i}+|x|^{s} \sum_{i=0}^{\infty}\left(\frac{C}{2^{\beta}}\right)^{i}\left(\sqrt[7]{2^{s}}\right)^{i}\right),
\end{aligned}
$$


for all $x \in \mathbb{R}$. As in the first case, the conditions $r, s<7\left(\beta-\log _{2} C\right)$ imply the convergence of the last two series in (26). Therefore,

$$
\|f(x)-L(x)\| \leq \epsilon C\left(\frac{|x|^{r}}{2^{\beta}-C \sqrt[7]{2^{r}}}+\frac{|x|^{s}}{2^{\beta}-C \sqrt[7]{2^{s}}}\right) .
$$

Third case: $\gamma(x, y)=\epsilon\left(|x|^{r}|y|^{s}+|x|^{r+s}+|y|^{r+s}\right)$.

Likewise, we get

$$
\begin{aligned}
\|f(x)-L(x)\| & \leq \frac{C}{2^{\beta}} \Gamma(x, x)=\frac{C}{2^{\beta}} \sum_{i=0}^{\infty}\left(\frac{C}{2^{\beta}}\right)^{i} \gamma\left(\sqrt[7]{2^{i}} x, \sqrt[7]{2^{i}} x\right) \\
& =\frac{C}{2^{\beta}} \sum_{i=0}^{\infty}\left(\frac{C}{2^{\beta}}\right)^{i} 3 \epsilon\left|\sqrt[7]{2^{i}} x\right|^{r+s},
\end{aligned}
$$

for all $x \in \mathbb{R}$. On the other hand, the condition $r+s<7\left(\beta-\log _{2} C\right)$ entails the convergence of the last series in (27). As a consequence,

$$
\|f(x)-L(x)\| \leq 3 \epsilon C\left(\frac{|x|^{r+s}}{2^{\beta}-K \sqrt[7]{2^{r+s}}}\right) .
$$

Corollary 2. Let $X$ be a quasi- $\beta$-Banach space. Moreover, let $f: \mathbb{R} \rightarrow X$ be a function such that

$$
\left\|f\left(\sqrt[7]{x^{7}+y^{7}}\right)-f(x)-f(y)\right\| \leq \begin{cases}\epsilon\left(|x|^{r}|y|^{s}\right), & r+s>7\left(\beta+\log _{2} C\right), \\ \epsilon\left(|x|^{r}+|y|^{s}\right), & r, s>7\left(\beta+\log _{2} C\right), \\ \epsilon\left(|x|^{r}|y|^{s}+|x|^{r+s}+|y|^{r+s}\right), & r+s>7\left(\beta+\log _{2} C\right),\end{cases}
$$

for all $x, y \in \mathbb{R}$, with $r, s, \in \in \mathbb{R}_{\geq 0}$. Then $E q$. (9) has a unique septic solution $L: \mathbb{R} \rightarrow X$ satisfying

$$
\|f(x)-L(x)\| \leq \begin{cases}\frac{\epsilon C}{\sqrt[7]{2^{r+s}}-2^{\beta} C}|x|^{r+s}, & r+s>7\left(\beta+\log _{2} C\right), \\ \epsilon C\left(\frac{|x|^{r}}{\sqrt[7]{2^{r}}-2^{\beta} C}+\frac{|x|^{s}}{\sqrt[7]{2^{s}}-2^{\beta} C}\right), & r, s>7\left(\beta+\log _{2} C\right), \\ \frac{3 \epsilon C}{\sqrt[7]{2^{r+s}}-2^{\beta} C}|x|^{r+s}, & r+s>7\left(\beta+\log _{2} C\right),\end{cases}
$$

for all $x \in \mathbb{R}$.

Proof. Following the same notation of Theorem 2, we have

$$
\gamma(x, y)= \begin{cases}\epsilon\left(|x|^{r}|y|^{s}\right), & r+s>7\left(\beta+\log _{2} C\right), \\ \epsilon\left(|x|^{r}+|y|^{s}\right), & r, s>7\left(\beta+\log _{2} C\right), \\ \epsilon\left(|x|^{r}|y|^{s}+|x|^{r+s}+|y|^{r+s}\right), & r+s>7\left(\beta+\log _{2} C\right),\end{cases}
$$

for all $x, y \in \mathbb{R}$. The rest of the proof runs as in Corollary 1 . 
Remark 2. Corollaries 1 and 2 show that $7\left(\beta \pm \log _{2} C\right)$ play a relevant role in the stability of functional Eq. (9). In particular, it is worth noticing that $\beta-\log _{2} C \leq 1$ and $\beta+\log _{2} C>0$. Accordingly, this means that functional Eq. (9) has two septic solutions in ]0,7], each of them is unique in the sense of (23), (24) and (28), (29).

\subsection{A counterexample of nonstability}

We now provide an example showing the nonstability of Eq. (9) in Corollary 1. More precisely, we prove that Eq. (9) is not stable for $r=s=7$ with $\beta=C=1$ for $\gamma(x, y)=\epsilon\left(|x|^{r}+|y|^{s}\right)$. Note that in (23) the conditions $r, s<7\left(\beta-\log _{2} C\right)$ do not hold, thus making the solution unstable.

Remark 3. Under the hypotheses of Theorem 1, Eq. (9) implies that

$$
f\left(q^{\frac{n}{7}} x\right)=q^{n} f(x), \quad q \in \mathbb{Q}, n \in \mathbb{Z}
$$

for all $x \in \mathbb{R}$. If moreover $f$ is continuous on $\mathbb{R}$, we have that $f(x)=x^{7} f(1)$ for all $x \in \mathbb{R}$.

Example 1. Let $\varphi: \mathbb{R} \rightarrow \mathbb{R}$ be a function defined as follows:

$$
\varphi(x)= \begin{cases}\alpha, & x \geq 1 \\ \alpha x^{7}, & |x|<1 \\ -\alpha, & x \leq-1\end{cases}
$$

with $\alpha>0$ being a constant. Moreover, let $f: \mathbb{R} \rightarrow \mathbb{R}$ be a function such that

$$
f(x):=\sum_{k=0}^{\infty} \frac{\varphi\left(2^{k} x\right)}{2^{7 k}},
$$

for all $x \in \mathbb{R}$. Under the above assumptions, $f$ satisfies the following inequality

$$
\left|f\left(\sqrt[7]{x^{7}+y^{7}}\right)-f(x)-f(y)\right| \leq \frac{3 \alpha \cdot 2^{21}}{127}\left(|x|^{7}+|y|^{7}\right),
$$

for all $x, y \in \mathbb{R}$ but in general there is no septic function $L: \mathbb{R} \rightarrow \mathbb{R}$ such that

$$
|f(x)-L(x)| \leq \lambda|x|^{7},
$$

for all $x \in \mathbb{R}$, with $\lambda>0$ being a constant.

First, note that the boundness of $f$ follows from (30). In fact, $|\varphi(x)| \leq \alpha$ for all $x \in \mathbb{R}$ implies that $|f(x)| \leq \frac{128}{127} \alpha$ on the entire real line.

We now proceed to prove that $f$ satisfies inequality (31). Obviously, (31) holds for $x=y=0$. Now we distinguish two case. For $|x|^{7}+|y|^{7} \geq \frac{1}{2^{7}}$ we see that

$$
\left|f\left(\sqrt[7]{x^{7}+y^{7}}\right)-f(x)-f(y)\right| \leq 3 \alpha \frac{128}{127}
$$


Thus (31) holds in $|x|^{7}+|y|^{7} \geq \frac{1}{2^{7}}$. Now suppose that $0<|x|^{7}+|y|^{7}<\frac{1}{2^{7}}$. It is straightforward to see that there is a $n \in \mathbb{N}_{0}$ such that

$$
\frac{1}{2^{7(n+2)}} \leq|x|^{7}+|y|^{7}<\frac{1}{2^{7(n+1)}}
$$

This implies that $2^{7 n}|x|^{7}<\frac{1}{2^{7}}$ and $2^{7 n}|y|^{7}<\frac{1}{2^{7}}$. Thus,

$$
\left.\left.2^{k} \sqrt[2^{k} x]{2^{k} y}\right\} \in\right]-1,1[, \quad k=0,1, \cdots, n-1,
$$

and so

$$
\varphi\left(2^{k} \sqrt[7]{x^{7}+y^{7}}\right)-\varphi\left(2^{k} x\right)-\varphi\left(2^{k} y\right)=0, \quad k=0,1, \cdots, n-1
$$

By (33), we have that

$$
\begin{aligned}
\left|f\left(\sqrt[7]{x^{7}+y^{7}}\right)-f(x)-f(y)\right| & =\left|\sum_{k=0}^{\infty} \frac{\varphi\left(2^{k} \sqrt[7]{x^{7}+y^{7}}\right)}{2^{7 k}}-\sum_{k=0}^{\infty} \frac{\varphi\left(2^{k} x\right)}{2^{7 k}}-\sum_{k=0}^{\infty} \frac{\varphi\left(2^{k} y\right)}{2^{7 k}}\right| \\
& \leq \sum_{k=0}^{\infty} 2^{-7 k}\left|\varphi\left(2^{k} \sqrt[7]{x^{7}+y^{7}}\right)-\varphi\left(2^{k} x\right)-\varphi\left(2^{k} y\right)\right| \\
& \leq 3 \alpha \sum_{k=n}^{\infty} 2^{-7 k} \leq \frac{3 \alpha \cdot 2^{21}}{127}\left(|x|^{7}+|y|^{7}\right),
\end{aligned}
$$

for all $x, y \in \mathbb{R}$ such that $0<|x|^{7}+|y|^{7}<\frac{1}{2^{7}}$. Therefore, we conclude that $f$ satisfies inequality (31) for all $x, y \in \mathbb{R}$.

We only need to show that Eq. (9) is not stable for $r=s=7$ and $\beta=C=1$ for $\gamma(x, y)=\epsilon\left(|x|^{r}+|y|^{s}\right)$ with $r, s<7\left(\beta-\log _{2} C\right)$. Suppose that there is a septic function $L: \mathbb{R} \rightarrow \mathbb{R}$ for which (32) holds. Note that continuity of $f$ on the entire real line implies that $L$ is continuous at the origin. Likewise, the boundness of $f$ on $\mathbb{R}$ entails that $L$ is bounded in any open interval containing the origin. Thus $L(x)=a x^{7}$ for any $x \in \mathbb{R}$ and for a suitable constant $a \in \mathbb{R}$, which follows from Remark 3 . As a consequence,

$$
|f(x)| \leq(\lambda+|a|)|x|^{7}
$$

for all $x \in \mathbb{R}$. On the other hand, we can take $l \in \mathbb{N}_{0}$ such that $\alpha l>\lambda+|a|$. It is worth noting that $\left.x \in\right] 0,2^{-l}[$ implies that $\left.2^{k} x \in\right] 0,1[$ for all $k=0,1, \cdots, l-1$. Accordingly,

$$
\left.|f(x)| \geq \sum_{k=0}^{l-1} \frac{\alpha\left(2^{k} x\right)^{7}}{2^{7 k}}=\alpha l x^{7}>(\lambda+|a|)|x|^{7}, \quad x \in\right] 0,2^{-l}[.
$$

This leads to a contradiction with (34).

\section{Hyers-Ulam stability of Eq. (9) in $(\beta, p)$-Banach spaces}

In this section, we generalize the Hyers-Ulam stability of Eq. (9) in $(\beta, p)$-Banach spaces. More precisely, we state and prove certain stability properties of Eq. (9) in these function spaces. 
First, we need to recall the notion of subhomogeneous function and superhomogeneous function. We refer the reader to $[35,36]$ for further information on the these definitions and related results.

Fix $d, \lambda \in \mathbb{N}$ such that $\lambda>1$. Let $\rho: X \rightarrow Y$ be a function with domain $X$ and codomain $(Y, \leq)$. Moreover, assume that $X$ and $Y$ are both closed under addition. We say that $\rho$ is a contractively subhomogeneous function of degree $d$ if there is a real constant $\theta$ such that

$$
\rho(\lambda x) \leq \theta \lambda^{d} \rho(x), \quad 0<\theta<1, x \in X .
$$

Likewise, we say that $\rho$ is an expansively superhomogeneous function of degree $d$ if there is a real constant $\theta$ such that

$$
\rho(\lambda x) \geq \frac{\lambda^{d}}{\theta} \rho(x), \quad 0<\theta<1, x \in X
$$

Note that removing the constant $\theta$ in Definition (35) and taking the equality sign, we get the well known definition of homogeneous function of degree $d$. In the literature, contractively subhomogeneous (resp. expansively superhomogeneous) functions of degree 1 are simply called contractively subhomogeneous (resp. expansively superhomogeneous) functions.

Remark 4. Let $l \in\{-1,1\}$. We see at once that

$$
\rho\left(\lambda^{\ln x}\right) \leq\left(\lambda^{l} \theta\right)^{n} \rho(x), \quad n \in \mathbb{N}, x \in X,
$$

if $\rho$ is either contractively subhomogeneous $(l=1)$ or expansively superadditive $(l=-1)$.

Now, we are in position to characterize the Hyers-Ulam stability of Eq. (9) in ( $\beta, p)$-Banach spaces, as stated in the following theorems.

Theorem 4. Let $X$ be $a(\beta, p)$-Banach space and $f: \mathbb{R} \rightarrow X$ be a $\gamma$-approximately radical septic function. Moreover, suppose that $\gamma$ is contractively subhomogeneous in the sense of (35) with $2^{1-7 \beta} \theta<1$. Then Eq. (9) has a unique septic solution $L: \mathbb{R} \rightarrow X$ satisfying

$$
\|f(x)-L(x)\| \leq \frac{\widehat{\Gamma}_{7}(x)}{\sqrt[p]{2^{7 \beta p}-(2 \theta)^{p}}},
$$

for all $x \in \mathbb{R}$, with

$$
\widehat{\Gamma}_{n}(x):=2^{7 \beta} \sum_{i=1}^{n}\left(\frac{C}{2^{\beta}}\right)^{i} \gamma\left(\sqrt[7]{2^{i-1}} x, \sqrt[7]{2^{i-1}} x\right)
$$

Proof. First, (17) implies that

$$
\left\|f(x)-\frac{f(2 x)}{2^{7}}\right\| \leq \frac{\widehat{\Gamma}_{7}(x)}{2^{7 \beta}} .
$$


Note that $\widehat{\Gamma}_{7}$ is contractively subhomogeneous in the sense of (35) with $2^{1-7 \beta} \theta<1$. Thus, replacing $x$ by $2^{i} x$ in (39), we have

$$
\begin{aligned}
\left\|\frac{f\left(2^{l} x\right)}{2^{7 l}}-\frac{f\left(2^{m} x\right)}{2^{7 m}}\right\|^{p} & =\left\|\sum_{i=l}^{m-1}\left(\frac{f\left(2^{i} x\right)}{2^{7 i}}-\frac{f\left(2^{i+1} x\right)}{2^{7(i+1)}}\right)\right\|^{p} \leq \sum_{i=l}^{m-1} \frac{1}{2^{7 i \beta p}}\left\|f\left(2^{i} x\right)-\frac{f\left(2^{i+1} x\right)}{2^{7}}\right\|^{p} \\
& \stackrel{(39)}{\leq} \frac{1}{2^{7 \beta p}} \sum_{i=l}^{m-1} \frac{1}{2^{7 i \beta p}} \widehat{\Gamma}_{7}\left(2^{i} x\right)^{p} \leq\left(\frac{\widehat{\Gamma}_{7}(x)}{2^{7 \beta}}\right)^{p} \sum_{i=l}^{m-1}\left(2^{1-7 \beta} \theta\right)^{i p}, \quad m, l \in \mathbb{N}_{0}: m>l,
\end{aligned}
$$

for all $x \in \mathbb{R}$. It is worth noting that $\left\{2^{-7 k} f\left(2^{k} x\right)\right\}$ is a Cauchy sequence. Since $X$ is a $(\beta, p)$-Banach space $X$, the previous sequence is convergent over the entire real line. This allows us to define a function $L: \mathbb{R} \rightarrow X$ by

$$
L(x):=\lim _{k \rightarrow \infty} \frac{f\left(2^{k} x\right)}{2^{7 k}}
$$

for all $x \in \mathbb{R}$. Hence

$$
\left\|L\left(\sqrt[7]{x^{7}+y^{7}}\right)-L(x)-L(y)\right\|^{p} \leq \gamma(x, y)^{p} \lim _{k \rightarrow \infty}\left(2^{1-7 \beta} \theta\right)^{k p}=0,
$$

and so

$$
L\left(\sqrt[7]{x^{7}+y^{7}}\right)=L(x)+L(y)
$$

for all $x, y \in \mathbb{R}$. From (41), we see that $L$ is a septic function. Moreover, letting $k \rightarrow \infty$ in (40) with $l=0$, we deduce that $L$ satisfies (37) near $f$.

Now, we proceed to show the uniqueness of $L$. Let $S: \mathbb{R} \rightarrow X$ be a septic function which also satisfies both (9) and (37). From (9), we see that $S\left(\sqrt[7]{2^{k}} x\right)=2^{k} S(x) \Rightarrow S\left(2^{k} x\right)=2^{7 k} S(x)$ for all $x \in \mathbb{R}$ and $k \in \mathbb{N}_{0}$. Accordingly, the contractively subhomogeneity of $\widehat{\Gamma}_{7}$ gives

$$
\left\|\frac{f\left(2^{k} x\right)}{2^{7 k}}-S(x)\right\|^{p} \leq \frac{\widehat{\Gamma}_{7}\left(2^{k} x\right)^{p}}{2^{7 \beta p}-(2 \theta)^{p}} 2^{-7 \beta k p} \leq \frac{\widehat{\Gamma}_{7}(x)^{p}}{2^{7 \beta p}-(2 \theta)^{p}}\left(2^{1-7 \beta} \theta\right)^{k p},
$$

which for $k \rightarrow \infty$ gives $S(x)=L(x)$ for all $x \in \mathbb{R}$. The proof is complete.

Theorem 5. Let $X$ be a $(\beta, p)$-Banach space and $f: \mathbb{R} \rightarrow X$ be a $\gamma$-approximately radical septic function. Moreover, suppose that $\gamma$ is expansively superhomogeneous in the sense of (36) with $2^{7 \beta-1} \theta<1$. Then Eq. (9) has a unique septic solution $L: \mathbb{R} \rightarrow X$ satisfying

$$
\|f(x)-S(x)\| \leq \frac{\widehat{\Gamma}_{7}(x)}{\sqrt[p]{\left(2 \theta^{-1}\right)^{p}-2^{7 \beta p}}}
$$

with $\widehat{\Gamma}_{7}$ defined as in (38) and for all $x \in \mathbb{R}$.

Proof. The theorem can be handled in the same way of Theorem 4. In fact, (39) entails that

$$
\left\|f(x)-2^{7} f\left(\frac{x}{2}\right)\right\| \leq \widehat{\Gamma}_{7}\left(\frac{x}{2}\right)
$$


for all $x \in \mathbb{R}$. Analysis similar to that in the proof of Theorem 4 shows that

$$
\begin{aligned}
\left\|2^{7 l} f\left(2^{-l} x\right)-2^{7 m} f\left(2^{-m} x\right)\right\|^{p} & =\left\|\sum_{i=l}^{m-1}\left(2^{7 i} f\left(2^{-i} x\right)-2^{7(i+1)} f\left(2^{-(i+1)} x\right)\right)\right\|^{p} \\
& \leq \sum_{i=l}^{m-1}\left\|2^{7 i} f\left(2^{-i} x\right)-2^{7(i+1)} f\left(2^{-(i+1)} x\right)\right\|^{p} \\
& \leq \sum_{i=l}^{m-1} 2^{7 i \beta p}\left\|f\left(2^{-i} x\right)-2^{7} f\left(2^{-(i+1)} x\right)\right\|^{p} \\
& \stackrel{(42)}{\leq} \sum_{i=l}^{m-1} 2^{7 i \beta p} \widehat{\Gamma}\left(2^{-(i+1)} x\right)^{p} \leq\left(\frac{\widehat{\Gamma}(x)}{2^{7 \beta}}\right)^{p} \sum_{i=l+1}^{m}\left(2^{7 \beta-1} \theta\right)^{i p}, \quad m, l \in \mathbb{N}_{0}: m>l,
\end{aligned}
$$

for all $x \in \mathbb{R}$. Thus $\left\{2^{7 k} f\left(2^{-k} x\right)\right\}$ is a Cauchy sequence. Taking into account the expansively superhomogeneity of $\widehat{\Gamma}_{7}$, the rest of the proof runs as in Theorem 4 .

The proof of Theorem 4 enables us to prove the Hyers-Ulam stability for Eq. (9) if $\gamma$ is contractively subhomogeneous (or expansively superhomogeneous) of degree $d>1$, as stated in the following two theorems.

Theorem 6. Let $X$ be $a(\beta, p)$-Banach space and $f: \mathbb{R} \rightarrow X$ be a $\gamma$-approximately radical septic function. Moreover, suppose that $\gamma$ is contractively subhomogeneous of degree 2 in the sense of (35) with $2^{2-7 \beta} \theta<1$. Then Eq. (9) has a unique septic solution $L: \mathbb{R} \rightarrow X$ satisfying

$$
\|f(x)-L(x)\| \leq \frac{\widehat{\Gamma}_{7}(x)}{\sqrt[p]{2^{7 \beta p}-(4 \theta)^{p}}}
$$

with $\widehat{\Gamma}_{7}$ defined as in (38) and for all $x \in \mathbb{R}$.

Theorem 7. Let $X$ be $a(\beta, p)$-Banach space and $f: \mathbb{R} \rightarrow X$ be a $\gamma$-approximately radical septic function. Moreover, suppose that $\gamma$ is is expansively superhomogeneous of degree 2 in the sense of (36) with $2^{7 \beta-2} \theta<1$. Then Eq. (9) has a unique septic solution $L: \mathbb{R} \rightarrow X$ satisfying

$$
\|f(x)-L(x)\| \leq \frac{\widehat{\Gamma}_{7}(x)}{\sqrt[p]{\left(4 \theta^{-1}\right)^{p}-2^{7 \beta p}}}
$$

with $\widehat{\Gamma}_{7}$ defined as in (38) and for all $x \in \mathbb{R}$.

Author Contributions: These two authors contribute equally to this paper. All authors have read and agreed to the published version of the manuscript.

Funding: The authors have not received funds for covering the costs to publish in open access.

Conflicts of Interest: The authors declare no conflict of interest. 


\section{References}

1. Ulam, S.M. Problems in Modern Mathematics; John Wiley \& Sons: New York, NY, USA, 1964; pp. $63-69$.

2. Hyers, D.H. On the stability of the linear functional equation. Proc. Natl. Acad. Sci. USA 1941, 27, $222-224$. [CrossRef] [CrossRef]

3. Aoki, T. On the stability of the linear transformation in Banach spaces. J. Math. Soc. Jpn. 1950, 2, 64-66. [CrossRef] [CrossRef]

4. Bourgin, D.G. Classes of transformations and bordering transformations. Bull. Am. Math. Soc. 1951, 57, $223-237$. [CrossRef] [CrossRef]

5. Rassias, T.M. On the stability of the linear mapping in Banach spaces. Proc. Am. Math. Soc. 1978, 72, $297-300$. [CrossRef] [CrossRef]

6. Rassias, J.M. On approximation of approximately linear mappings by linear mappings. J. Funct. Anal. 1982, 46, 126-130. [CrossRef] [CrossRef]

7. Forti, G.L. The stability of homomorphisms and amenability, with applications to functional equations. $A b h$. Math. Semin. Univ. Hambg. 1987, 57, 215-226. [CrossRef] [CrossRef]

8. Găvruţa, P. A generalization of the Hyers-Ulam-Rassias stability of approximately additive mappings. J. Math. Anal. Appl. 1994, 184, 431-436. [CrossRef] [CrossRef]

9. Brzdęk, J.; Ciepliński, K.; Leśniak, Z. On Ulam's type stability of the linear equation and related issues. Discret. Dyn. Nat. Soc. 2014, 2014, 536791. [CrossRef] [CrossRef]

10. Brzdęk, J.; Cădariu, L.; Ciepliński, K. Fixed point theory and the Ulam stability. J. Funct. Spaces 2014, $2014,829419$. [CrossRef] [CrossRef]

11. Cădariu, L.; Găvruţa, L.; Găvruţa, P. Fixed points and generalized Hyers-Ulam stability. Abstr. Appl. Anal. 2012, 2012, 712743. [CrossRef] [CrossRef]

12. Bahyrycz, A.; Brzdęk, J.; Jabłońska, E.; Olko, J. On functions that are approximate fixed points almost everywhere and Ulam's type stability. J. Fixed Point Theory Appl. 2015, 17, 659-668. [CrossRef] [CrossRef]

13. Chang, I.S.; Jung, Y.S. Stability for the functional equation of cubic type. J. Math. Anal. Appl. 2007, 334, 85-96. [CrossRef] [CrossRef]

14. Lee, S.H.; Im, S.M.; Hwang, I.S. Quartic functional equations. J. Math. Anal. Appl. 2005, 307, 387-394. [CrossRef] [CrossRef]

15. Lee, Y.H. On the Hyers-Ulam-Rassias stability of a general quintic functional equation and a general sextic functional equation. Mathematics 2019, 7, 510. [CrossRef] [CrossRef]

16. Shen, Y.; Chen, W. On the stability of septic and octic functional equations. J. Comput. Anal. Appl. 2015, 18, 277-290. [CrossRef]

17. Almahalebi, M.; Charifi, A.; Park, C.; Kabbaj, S. Hyperstability results for a generalized radical cubic functional equation related to additive mapping in non-Archimedean Banach spaces. J. Fixed Point Theory Appl. 2018, 20, 40. [CrossRef] [CrossRef]

18. EL-Fassi, Iz. A new type of approximation for the radical quintic functional equation in non-Archimedean (2, $\beta$ )-Banach spaces. J. Math. Anal. Appl. 2018, 457, 322-335. [CrossRef] [CrossRef]

19. Eshaghi Gordji, M.; Khodaei, H.; Ebadian, A.; Kim, G.H. Nearly radical quadratic functional equations in p-2-normed spaces. Abstr. Appl. Anal. 2012, 2012, 896032. [CrossRef]

20. Khodaei, H.; Eshaghi Gordji, M.; Kim, S.S.; Cho, Y.J. Approximation of radical functional equations related to quadratic and quartic mappings. J. Math. Anal. Appl. 2012, 395, 284-297. [CrossRef] [CrossRef]

21. Kim, S.S.; Cho, Y.J.; Eshaghi Gordji, M. On the generalized Hyers-Ulam-Rassias stability problem of radical functional equations. J. Inequal. Appl. 2012, 2012, 186. [CrossRef] [CrossRef]

22. Cho, Y.J.; Gordji, M.E.; Kim, S.S. On the stability of radical functional equations in quasi- $\beta$-normed spaces. Bull. Korean Math. Soc. 2014, 51, 1511-1525. [CrossRef] [CrossRef]

23. Ding, Y.; Xu, T.Z. Approximate solution of generalized inhomogeneous radical quadratic functional equations in 2-Banach spaces. J. Inequal. Appl. 2019, 2019, 31. [CrossRef] [CrossRef] 
24. EL-Fassi, I.I. Solution and approximation of radical quintic functional equation related to quintic mapping in quasi- $\beta$-Banach spaces. RACSAM 2019, 113, 675-687. [CrossRef] [CrossRef]

25. EL-Fassi, I. New stability results for the radical sextic functional equation related to quadratic mappings in in (2, $\beta$ )-Banach spaces. J. Fixed Point Theory Appl. 2018, 20, 138. [CrossRef] [CrossRef]

26. Alizadeh, Z.; Ghazanfari, A.G. On the stability of a radical cubic functional equation in quasi- $\beta$-spaces. J. Fixed Point Theory Appl. 2016, 18, 843-853. [CrossRef] [CrossRef]

27. EL-Fassi, I.I. Approximate solution of radical quartic functional equation related to additive mapping in 2-Banach spaces. J. Math. Anal. Appl. 2015, 455, 2001-2013. [CrossRef] [CrossRef]

28. El-hady, E.; Brzdȩk, J.; Nassar, H. On the structure and solutions of functional equations arising from queueing models. Aequ. Math. 2017, 91, 445-477. [CrossRef] [CrossRef]

29. Guillemin, F.; Knessl, C.; van Leeuwaarden, J.S.H. Wireless multihop networks with stealing: large buffer asymptotics via the ray method. SIAM J. Appl. Math. 2011, 71, 1220-1240. [CrossRef] [CrossRef]

30. Balibrea, F.; Reich, L.; Smítal, J. Iteration theory: Dynamical systems and functional equations. Int. J. Bifur. Chaos Appl. Sci. Engrgy 2003, 13, 1627-1647. [CrossRef] [CrossRef]

31. Sahoo, P.K.; Székelyhidi, L. On a functional equation related to digital filtering. Aequ. Math. 2001, 62, $280-285$. [CrossRef] [CrossRef]

32. Benyamini, Y.; Lindenstrauss, J. Geometric Nonlinear Functional Analysis; American Mathematical Society: Providence, RI, USA, 2000; pp. 445-447.

33. Wilansky, A. Modern Methods in Topological Vector Spaces; McGraw-Hill: New York, NY, USA, 1978 ; pp. 18-24.

34. Rolewicz, S. Metric Linear Spaces; PWN \& D. Reidel: Warsaw, Poland, 1984; pp. 95-96.

35. Eshaghi, M.; Abbaszadeh, S. Approximate generalized derivations close to derivations in Lie $C^{*}$-algebras. $J$. Appl. Anal. 2015, 21, 37-43. [CrossRef] [CrossRef]

36. Gordji, M.E.; Khodaei, H. A fixed point technique for investigating the stability of $(\alpha, \beta, \gamma)$-derivation on Lie $C^{*}$-algebras. Nonlinear Anal. Theory Methods Appl. 2013, 20, 52-57. [CrossRef] [CrossRef]

Publisher's Note: MDPI stays neutral with regard to jurisdictional claims in published maps and institutional affiliations.

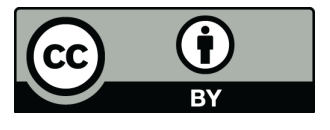

(C) 2020 by the authors. Licensee MDPI, Basel, Switzerland. This article is an open access article distributed under the terms and conditions of the Creative Commons Attribution (CC BY) license (http:/ / creativecommons.org/licenses/by/4.0/). 